\title{
In the footsteps of Einstein and Wiener
}

\section{I}

At any time and era, science has always been evolving in two trends: integrational and reductionist. Both of these alternative vectors determine (regardless of whether we want it or not) a development path of the entire intellectual world community. But then all of a sudden a very unexpected study appears, in which the above vectors are creatively intertwined, yielding ultimately something out of the ordinary!..

It is from such positions that one can, apparently, consider publishing of the wellknown books "Synergetics” by Herman Haken, “A mathematical theory of communication” by Claude Shannon, "Philosophy and computing" by Luciano Floridi, "Proof of the simulation argument” by Nick Boström. Although, as it turned out later, they all had more respectable and far-sighted Eastern predecessors (Ex oriente lux. - Sic!). First and foremost, this is, of course, a truly legendary figure A.A. Bogdanov-Malinovsky (invented tektology); pioneer of Soviet relay-contact circuits V.I. Shestakov; the distinguished domestic cosmoglobalist A.D. Ursul. It was the Vice-president of "Moldavian academy of sciences" Arkady Ursul, who managed to formulate a necessary cybernetic criterion of evolutionary development and also proposed a bold advanced hypothesis on the role of information in improvement of the surrounding world in general and social progress in particular.

As for the computer-simulated theory, which is popular nowadays, it first appeared in the works of the modern Ukrainian naturalist Y. N. Teslia, not in frigid-rocky Scandinavia.

Are you intrigued by so suddenly brisk start? Then we dare to assure you that not only one, but exactly five the freshest innovative ideas will be presented to all readers, who are not indifferent to the search for truth, in this multidisciplinary research paper. Which could literally turn, among other things, universally accepted scientific fundamentals upside down! And moreover author's arguments are supported with specific physical \& mathematical calculations or corresponding formulas as far as possible.

What is information? Throughout the last century, this seemingly_lowbrow question has stubbornly haunted many learned minds that as a result led to the emergence of diverse and sometimes exotic views on its nature, laws and properties. Though contingently, all these theories can still be divided in two principal subgroups. At that the first (more early) includes those definitions, where information was considered as a kind of applied hypostasis of everyday human life. Such standpoint was particularly shared by Norbert Wiener, meaning in that case "a name for the content of what is exchanged with the outer world as we adjust to it". However, now the vast majority of researchers (following Arkady Ursul \& Luciano Floridi) have eventually come to the output that information is a quite objective category inherent in any material body, not only animate. And that's exactly another, more advanced stage of the progressive semantic development of this concept. Though all similar inferences are based on the understanding of information as a measure of orderliness. For its part, the latter is created by the telic activity of a person, a robot, or - at an elementary level 
- some really existing “informons". At that all informons must a priori have a short-term memory, which they learned (in the course of own evolution) to translate into a long-term. But, a true, already with the help of other, more massive and instrumentally observed particles. Thus, any information - even human or computer (presented here as if summarizedly) - is "nurtured” and brought by informons.

As for the ill-fated but so many-sided chaos, on that issue the scientific community has been sharply divided at all. If, on the one hand, the majority of "techies" (linking to the first and the second law of thermodynamics) consider it a real factor, then already their antipodes-humanities are clearly inclined to classify it like a purely residual phenomenon which just indicates absence of order. Proposing an obvious analogy, in particular, with a scrawny disheveled Death who can exist except that in someone's morbid imagination or on frightening pictures from old church calendars. However, be that as it may, any purposeful human actions for sure - at least either by a iota, at least or by bit - reduce Universe's entropy. I.e. in practice, each man-made change creates a certain countable information product (even if it's as imperceptibly small as Planck quantum of time), distancing us from the notorious doomsday. But still in more detail we'll talk about that in final sections of this article.

\section{II.}

According to the generally accepted version, everything in the world began from singular clot of proto-energy (prāna) which had zero information. Nowadays the residual energy is clearly in obedience to the Supreme Will (although other subordinate relations are quite possible between them too). At the same time, all alone it is apparently not capable of creating new information, but yet there is a high probability that knows how to absorb ready-made one (with the aim of ensuing conversion to energy).

And on the whole, you can, by and large, imagine three basic dynamic schemes of universe:

a) a sort of swing "from energy (Will) $\Rightarrow$ to information (Reason) and back";

b) continuous experiments or even improvisations of Will itself (by the by, the old theosophical teaching about the previously existed 5 discarnate races also fits into here);

c) and finally, as if independently of them, the aforementioned hypothesis about our being qua computer simulation of the physical world can be considered too (moreover, in this case, bits and bytes well familiar to IT-specialists become already the main source of interaction of all real \& virtual objects).

As for the Supreme Reason, it (in contrast to the articulate canons of the Christian trinity) does not have any definitions like "Information-the-Mother”* (which, in principle, should be identical to Universe's noosphere), “Order-the-Son” or "the Holy Negentropy”. And even the existence of a number of rapid-fire mnemonists (Yuri Gorny, Andriy Slyusarchuk, Shakuntala Devi) as well as phenomenal clairvoyants (Michel de Nostredame, Edgar Cayce, Vangelia Gushterova) is not, alas, any weighty and convincing argument in 
sense of their involvement in such a constellation of "orderly sons". For the alternative version (concerning the usage of hidden brain resources) appears more preferred here. Although, along with this, the quite obvious fact that it is precisely the negentropy scattered everywhere (or rather - its elementary carriers) plays a decisive role in planetary and technological progress, is now perceived, in essence, as an indisputable truth.

In any non-artificial (i.e. created by nature itself) objects, the information - as, say, rudimentary short-term memory - is capable of circulating for a certain period of time inside closed organic rings. And even more so, if such rings pack (as, for example, in the structure of polynucleotides) one under the other into a three-dimensional perfectly coordinated chain, then apparently, this provides us serious grounds to assume that they have some elements of the psyche. Let alone the simplest semiconductor triodes handling the exterior (i.e. clearly brought from above) genetic code on a given algorithm.

Since both information and self-organization are directly related to orderliness, therefore, these are links of all the same Universe's process. The single difference here (and even then - rather, at the level of common terminology) is that informons were born immediately after the Big Bang, while real self-organization can be talked about only, perhaps, starting from the appearance of new auxiliary attributes connected with long-term memory.

That is why the notorious scientific thesis that "the Universe is streamlining in a qualitative (ie, more significant and important) plan but being disordered in a quantitative (it means heat entropy)" should, apparently, be extended towards all classical laws of thermodynamics (and particularly the second). Although the same applies to synergetics either - contrary to Ilya Prigogine's opinion (supposedly separate fluctuations arise against the overall increase in entropy, but only in some limited space).

That is, most likely, disordering goes along the energy vector, and ordering does the informational one. However this dependence is not linear in nature, for a developed intelligence creates new algorithmic products lot easier and faster - without high energy costs (as compared to that was at the dawn of becoming the Universe). And this means the dilemma "what exactly the current algorithms are generated by: self-organization or intelligence” doesn't already make much sense, because any contemporary intelligence is, in turn, a visual result of self-organization. Practically all the laws of physics, chemistry and biology that exist nowadays (and especially the first of mentioned) are consequence of the action of self-organizing processes, for all of them, somehow or other, are associated with motion.

By the way, in one of his recent monographs, the vice-rector of the metropolitan Aviation University Y.N. Teslia suggested the following equation comparing the speed of a moving material body with its information content:

$$
\vec{V}=(2 \cdot p-1) \cdot \vec{c}=\frac{d}{i} \cdot \vec{c}
$$

where $\vec{c}$ is the speed of light in vacuum; $\vec{V}$ - a drift velocity of the object; $p-$ probability of displacement of a given object by one quantum of space for one quantum of 
time; $d$ - quantitative predetermination of such a movement; $i$-complex awareness of the object about the potential dynamic capabilities (for each specific case).

Going back to the core idea, which appears in the article title, I'd like to note that this equation can be extrapolated (and most importantly, very successfully) to the Einstein's theory of special relativity in the modern scientific space of Norbert Wiener. Due to the fact it is directly based on the informational and probabilistic interpretation of mechanical movement.

That is, after a thorough examination, according to the conclusions of Yuri Nikolaevich, there is something in nature that we perceive under the guise of spatiotemporal dependence, universal gravitation, electromagnetism, weak and strong nuclear interactions. However, all these visible (although often deceptive) effects are realized through the corresponding info-fields. In other words, if some nth field exists, it means that somewhere there must be an object that creates it - which, according to prof. Teslia's logic, is already authentic information for other objects neighboring it. Therefore, I would like to cite another curious and bold quote from his most recent published works: "The motion of any natural body is determined by their information content, i.e. ability developed over 13.8 billion years to correctly respond to the existence of other neighbors. Especially taking into account that during such a long period of time a sufficient cluster of reflexes** useful for them has already appeared”.

\section{III.}

As it known, order and ordering are two different philosophical categories: the first is determined by a finite set of structural components in the post-singular phase; while the second is characterized by the existence of some tiny "super-hard-workers" which are constantly involved in the building a percentage of the order everywhere around them. At that the main functional advantage of such micro-agents is their memory. However, since we are still talking here about common elementary particles, then of course, they are very far from possessing truly constructive skills. By and large $e_{2}$ some initial creation glimpses (let alone planning) could arise exceptionally after their rallying in a limited volume. And even then - only under the influence of an extraneous factor, because they will be unlikely able to organize themselves into such a system.

At that regard, the trendy now but very controversial "law of indestructibility of karmic information”, apparently, would be more correct to formulate as follows: a complete intellectual product (made both by human community and the catchall cosmic supramental as well as scattered informons) cannot be, unfortunately, auto-saved. Meanwhile, how long successful fleeting notion-images or even priceless author's finds may keep in memory of the creators of such a product - that's really enough difficult issue solved every time in own way.

As for one more property of information that is no less relevant - virtually unlimited copyability - in theory, it should be determined by the corresponding characteristics of its 
elementary carriers; namely, either self-doubling, or contactless transmission of some internal parameters. Therein lays the main difference between such mobile and harmonious microparticles from the "Holy Spirit”, which (even if, for example, we'll identify it with a certain gauge field) is based on absolutely inert and weightless but, on the other hand, the eternal and truly all-pervading “volintons". In addition, it isn't excluded that the energy presented to us from above (exactly the same as the Universal Time) can't be broken down into the smallest discrete components at all, since is de-facto an attribute, not a subject of progressive evolutionary development (or, if you like, interplay between any types of matter).

By the by, seemingly polarity of interests of Reason and Will is actually sometimes felt except that, perhaps, in the socio-historical plane. While in all the rest, they go near, as if complementing each other; and moreover at the supremacy (for now, at least) of Reason. In this way, here it would be admissible (and even then - on Universe's scale) their principled opposing only in terms of time parameters: from the energy of saturated but structureless chaos - to an extremely structured but cold-lifeless Cosmos. And just at this finish segment, due to the critical shortage of natural resources, the energy-dependent transition to the hegemony of robots seems a quite real.

\section{IV.}

Since information and energy have a common starting point in their lineage, it means that some self-explanatory parallels aren't excluded between them. So, for example, by analogy with the classical Einstein's formula $\mathbf{E}=\mathbf{m c}^{2}$ (connecting rest mass with energy), it is possible, apparently, to make a similar equation for the needs of synergetics either. However, in this case, it should undoubtedly be taken into account that with the same weight a 6-ton pile of manure, an African elephant and the academic staff of a design institute will have completely different informational significatives (let alone citation indices). Moreover, even the aforementioned elephant itself can be either dead or alive; and the institute could located as in civilized Europe as amid the wild Papuan jungles (with the corresponding IQ of its employees). That is why here we should primarily operate with a hidden (i.e. dark matter) instead of the usual mass, given simultaneously that of five theosophical sheathes at least $90 \%$ of the information is concentrated exactly on the level of the mental and also within the field carcass (or so-called "spirit").

And yet, in spite of the apparent problematic nature, it's possible, in theory, to count such almost elusive visually substance. For this, it's quite enough just to deduct the "lion's share" of the nucleons from the total weight of a person. Although, by the way, there is another fallback method consisting in long-term (two- or even three-day) thorough observation of dying people, from which, in principle, the subtle posthumous entities of interest to us should separate one by one.

Further, instead of lightspeed we put, needless to say, the velocity of hypothetical informons propagation in a vacuum. 
And, finally, the turn came to the most, perhaps, difficult part of the task associated with inconspicuous but crucial in its role a superscript character. Indeed, despite the fact the level of organization of matter, it seems, should be present here, however, in what exactly form (coefficient, degree, natural logarithm or even factorial) - that's, alas, still unclear. Besides, the question remains open whether to take into account the intersystem levels (atom - molecule - nucleotide - gene - cell - family - nation) as self-substanted or to be limited to only five basic, spiritualized ones.

Thereby, the provisional formula of the immanent (synonyms: inward, apriorian) information intrinsic to a certain physical body could roughly look like this:

$$
\mathbf{I}_{(\mathbf{p})}=\left(\mathbf{M}_{(\mathbf{d})} \mathbf{K}\right)^{\mathbf{L}}
$$

(where L - a positive integer which in practice, i.e. excluding the infinite fractal divisibility in the spirit of Anaxagoras, can be in no wise greater than 15; so in this context the superior " $L$ " has nothing to do with the logarithm: it's just an abbreviation for the word "level”).

And concerning the long-term human memory, as the main repository of all acquired and newly created information, then foremost it is obviously important to find out, on what numeral system (robotized binary or more familiar for us decimal one) such a memory is recorded in the deep brain structures. By the way, in the first case, the sum of active mnemo-bits would probably be much easier to calculate - for example, by the total amount of significant dual "jumpers" in there protein molecules. But, true, given the fact that not all intrapeptide hookups can fulfill this role. In particular, center carbon stroma (together with carboxyl group directly attached to it) should be recognized as creatively inert organic foundations, while amino-pyramid and branched side radical are just the very carriers of memory that are of interest to us.

The power of thinking is determined almost similarly; but with the obligatory consideration, furthermore, the multi-tiering of "substantia grisea", due to what the cumulative number of possible relay switches as a result exponentially increases. At that it's both within the hemispheric modules themselves, and between individual links of the neoand paleocortex.

Nevertheless, it should still be emphasized that converting information into energy is objectively estimated (well, at the current stage of technical-engineering readiness, at least) as an completely illusory, arch-fantastic venture for modern mankind. And even their unified starting point is unlikely to somehow help here: after all, though by ontological pedigree they are indeed "sisters" but, alas, in no way twins. So the external similarity of the certain laws of development does not mean anything yet!..

\section{V.}

Weird artifacts on snapshots taken in pitch darkness (but with manual exposure), Raudiv's voices, "phonic modulations” by Lamoreau brothers as well as various heavenly and especially UFO-signs indicate either that interpreting by us information - as holistic 
panuniverse phenomenon - can be not quite correct, or we perceive it, and hence are aware through our subtle sheaths, i.e. warpedly. At the same time, the most prominent role in explaining such paradoxes belongs, perhaps, to modern American scientists J. Allen Hynek and Jacques Vallee who advanced own alternative hypothesis of extradimensional intelligence (abbr. - EDI) to the global academic community. In particular, thanks to their restlessness and ebullient exploratory enthusiasm, somewhere at the end of the last century, it became possible to put into overall use many demanded today by researchers the terms like sensory filter, info-subjectivism, parallel worlds. Although, as it thereafter turned out, even the very father of cybernetics Norbert Wiener had latent doubts about precise determinity of information, implying by it (in contrast to the rustic and artless cryptographer Shannon) enough vague data that any actively perceiving subject is able to interpret in his way.

But with that, despite the seeming speculativeness of this concept, it certainly must still have a specific material justification (at least in the form of some so far elusive quasiparticles or a gauge field); forasmuch otherwise one can sink to empty sophistry and agnosticism. So, to this end let's now refer to the law of over-cupple similarity*** and recall that the direct hierarchical ancestor of any multi-cellular organism is a free thermal neutron, alas, fairly limited in terms of its vital activity - as, unfortunately, the current "master of nature" Homo sapiens himself. It lives on average $880.1 \pm 1.1$ seconds, after that breaks down, as a rule, into three shards which are completely inequivalent. But since at times other exclusive cases can be observed here, it's better to visually represent all this as a triad of paired formulas. Paired - because next to each trivial quantum-mechanical equation, its macro-analogy projected, so to speak, on our reality will be together indicated too.

$$
\begin{gathered}
\text { a) } \beta^{1}\left(\mathrm{n} \rightarrow \mathrm{p}+\mathrm{e}^{-}+\bar{\vartheta}_{\mathrm{e}}\right) \\
\mathrm{LC} \rightarrow \mathrm{s} / \mathrm{d}+\mathrm{s}^{\mathrm{v}}+\mathrm{s}^{\mathrm{m}}
\end{gathered}
$$

Here is ordinary, i.e. quite ubiquitous type of death, in which a former living creature decomposes into a corpse (subject for dissection), a vegetative lepton covering and a residual mental sheath.

$$
\begin{aligned}
& \text { б) } \beta^{2}\left(\mathrm{n} \rightarrow \mathrm{p}+\mathrm{e}^{-}+\bar{\psi}_{\mathrm{e}}+\mathrm{\gamma}\right) \\
& \mathrm{LC} \rightarrow \mathrm{s} / \mathrm{d}+\mathrm{s}^{\mathrm{v}}+\mathrm{s}^{\mathrm{m}}+\mathrm{s} / \mathrm{s}
\end{aligned}
$$

And this is just more unusual variant - with the extra emission, besides, of weightless shining substance. Wherein, apparently, only some the most advanced gurus, prophets and wonderworkers are able to get out of life in this way.

$$
\text { в) } \begin{gathered}
\beta^{3}\left(\mathrm{n} \rightarrow{ }^{1} \mathrm{H}+\overline{\mathrm{v}}_{\mathrm{e}}\right) \\
\mathrm{LC} \rightarrow \mathrm{hr}+\mathrm{s}^{\mathrm{m}}
\end{gathered}
$$

And that is already the rarest, truly unique natural phenomenon, which results in the emergence of incorruptible holy relics - when the body still retains certain of its vegetative functions, although the person himself is irrevocably dead.

\section{Conclusion}


So, now is the time to draw a resumptive line, that is, to answer the question as clearly and easily as possible, what inferences useful for academic science could follow from here.

Well, to begin with the fact the sole carriers (and perhaps even the original creators) of information can, obviously, be except that amazingly ever-present, energetic and tireless hard workers-leptons. And besides this output is quite defined, specific, peremptory and is not, as they say, subject to appeal. For, no matter what global paradigm we here hold, but both in the expanded (so called Teslya's) formulation, and in the traditional one, only the smallest electron-neutrino ensembles contain everything truly valuable and meaningful for any natural system. Secondly, the resurrection of the dead (be it in a new-created or even former, seemed already outlived itself body - which is sometimes found out during the exhumation), it turns out, practically exists indeed. In modern nuclear physics, a similar process (the opposite - I note - in relation to all three of the above options) is, properly speaking, well studied too and named, by the by, scientifically as K- or L-capture. This is described more detail in the latest fundamental edition of the director of the metropolitan "Institute of artificial intelligence” acad. Anatoly Shevchenko "The path to Truth"».

But with that, as if along the way, many other purely corpuscular "inconsistencies" immediately also surface here. For example, at least following. As indicated in any university tutorial on cosmology, right there after the Big Bang, in a kind limited space an almost equal amount of currently known elementary particles (including protons, neutrons, antiprotons, electrons, positrons, etc.) were formed. However, due to direct contact of matter with antimatter, the lion's share of them has mutually annihilated, thereby giving a push to the ubiquitous relic radiation. And further (in the same textbook), literally a few pages later, we read: the total number of neutrons in the Universe now is about $15 \%$ of the corresponding one of protons. Wait, but how is this, pardon, possible: after all, they (being neutral per definitionem) did not react with anyone - which means that everything, by and large, should be exactly the opposite!.. So turns out, only authorian theory of spiroemanogenesis can offer a sufficiently intelligible consistent justification here: for, in essence, most of protons' inert mass is simply an elementary rubbish dump (or, if you like, an accumulation of corpses) left over from the former neutron vital activity. Because in the femto-world, alas, there aren't own bacteria or fungi that would be able to decompose carrion.

Nevertheless, as with any innovative concept, unfortunately, not without certain dark spots here. And the most obvious of them is somehow connected with an adequate interpretation of the very mysterious and truly elusive "volintons". So it remains for the time having to reassure myself that given philosophical issue is clearly just beyond the bounds of informatics. However, on the other hand, this also means that it will necessarily need to pay more close attention in our future publications!.. 
* But at the same time, it is the computer simulation theory that asserts fact such kind of "Information-the-Mother" must definitely somehow manifest itself in our world!

${ }^{* *}$ Here it's implied that reflexes present an integral response of this particular object to information came from the outside.

*** See the previous authorian article on this matter: «The evolution of natural systems - from the point of view of a philosopher»: https://openscience.uz/index.php/sciedu/article/view/ $\underline{1694}$

Emir E. Ashursky /Kiev, Ukraine/ 